\title{
Research on the Construction of Collaborative Training Technical Platform for Cultivation of Postgraduates of Professional Degree in Clinical Medicine Major and Standardized Training of Resident Doctors
}

\author{
Xiaoping Zhu, Gaofei Cao and Ke Yu*
}

\author{
Sichuan Medical University, Luzhou Sichuan, China 646000 \\ *Corresponding author
}

\begin{abstract}
Keywords: Degree of clinical medical science, Standardized training of resident doctors, Collaborative Training, Technical platform.
\end{abstract}

\begin{abstract}
The collaborative training platform for postgraduates of clinical medicine and standardized training of resident doctors is network service platform that provides resource sharing, remote teaching, research interaction and planning management for the education of postgraduates of clinical medicine and standardized training of resident doctors as well as a system formed by the mother and daughter platforms that connect to each other. The realization of the technical platform is based on multi-level distribution network system structure, distributed architecture technology and applied techniques such as Web, multimedia, databases, etc.
\end{abstract}

\section{Introduction}

On June 30, 2014, six departments including the Ministry of Education, the National Health and Family Planning Commission jointly issued Views on Deepening the Reform of Clinical Medical Personnel Training Based on Collaborative Medical Education (Teaching and Research [2014] No. 2). The document points out: "Since 2015, all new recruit clinical medicine graduate students of professional degree will be resident doctors that participate the standardized training of resident doctors. The clinical training conforms to the unified state requirements for standardized training of resident doctors ${ }^{[1]}$. This move taken by the State, in fact is to reform the training mode of clinical medical postgraduates of professional degree in China, aiming at realizing "combination of the three", i.e., of recruitment and admission, cultivation and training, and requirement of degree conferring and clinicians access system for the education of clinical medical postgraduates of professional degree and the training of resident doctors respectively, at making it fully align with to the commission of standardized training of resident doctors, and at establishing a system and mechanism of training clinical medical graduate students of professional degree in collaboration with standardized training of resident doctors (hereinafter referred to as "collaborative training") ${ }^{[2]}$.

This has brought a big challenge to postgraduate training units and departments of standardized training of resident doctors. In order to ensure the smooth implementation of the commission, it is required to establish related guarantee measures from all aspects. Firstly, educational resources should be further arranged among the State, provinces, postgraduate training units and departments of standardized training of resident doctors so as to enable them to be shared in various provinces and regions. At the same time, in order to guarantee students' clinical practice time of 33 months, graduate courses teaching, scientific research and dissertations will mainly be completed in spare time. Thus, it is in urgent need to build a network technical platform for the students to independently complete the task of learning and scientific research. Thereby, this paper proposes to build a network technical platform for collaborative training, which provides convenient network service for collaborative training of personnel, so as to achieve resources integration and sharing for 
education of clinical medical postgraduates of professional degree and standardized training of resident doctors.

\section{Functional Orientation of Collaborative Training Technical Platform}

The construction of the platform is oriented to realize integrated, interactive and hand in hand development of education of clinical medical postgraduates of professional degree and standardized training of resident doctors and integrated use of education resources among various educating and training units between and to avoid waste of resources.

From the perspective of integrated education, it aims at establishing a cultivating community of intergrowth, mutual integration, complementary chiasma and sustainable development between the educating units of clinical medical postgraduates of professional degree and the hospitals for standardized training of resident doctors and realizes deep integration and respective sustainable development of both by means of two-way flow and optimized allocation of educational resources and elements among various universities and training bases ${ }^{[3]}$. In this sense, it is an urgent need of medical personnel training and development to establish the platform under the background of "synergy of medical practice and education".

The platform mainly provides function such as resource sharing, remote teaching, research interaction and planning management. It possesses three major features. Firstly, it is service-oriented and based on SaaS (Short form of Software as a Service). That is, it is a technical platform that provides service to release postgraduate students from tedious task of teaching and learning, scientific research and clinical practice and to enable them to take full advantage of the convenient function of network and to put limited energy into endless learning. Secondly, it takes network as the carrier. The platform is neither a workbench in the traditional sense nor the ordinary environment or condition required for the respective development of medical practice and education, but the network environment necessary to "synergy of medical practice and education". It is a network platform based on the integration of content and technology, which can effectively break through the space and time limit of integration and sharing of health care and education resource and greatly enhance the efficiency of collaborative development. Thirdly, its major functions lie in resource sharing, remote teaching, research interaction and planning management, which are the embodiment of the overall arrangement making function of the collaborative training platform.

\section{Architecture of Collaborative Training Technical Platform}

From the perspective of management, the collaborative training technical platform is a system formed by a mother and a daughter platform based on and connected with each other. The mother platform is one that managed by the provincial education and health facilities, mainly responsible for the overall planning management of all the technical platforms of the cultivating communities within the scope of the province while the daughter platform is one that managed by the cultivating communities, mainly responsible for the planning management of educational resources within the communities. The former is of a one-to-many relationship with the latter. That is the mother platform controls more than one daughter ones. Besides, they are both interconnected and mutually independent. - a relationship mainly embodied in their specific categories.

Specific Categories of the Mother Platform. The mother platform covers seven modules, i.e., "Introduction to the Platform", "Medical Practice and Education Dynamic", "Cultivating Community", "Resource Sharing", "Distance Education", "Research Interaction" and "Planning Management". Among them, the first module focuses on introduction to basic situation such as the 
platform's functional localization and concrete contents. The second focuses on timely introduction to information of policies, examination, enrollment, employment, etc. related to medical practice and education. The third focuses on details of each training community, including the composition, work progress and effect of it. The next three modules lay emphasis on providing preliminary experience of them as well as the basic interface that links to the daughter platform, which is designed to enable users to get a comprehensive and vivid understanding of the three modules and to learn how to use them through experiencing. At the same time, they provide entry for the user to enter three corresponding daughter modules. The last focuses on maintenance of the mother platform and management of the daughter platform, designed to enable managers to better safeguard platform operation.

Specific Categories of the Daughter Platform. As mentioned earlier, the mother and daughter platforms are interrelated and independent of each other. Specifically, their interrelatedness lies in two aspects: one is direct access to the daughter platform through the mother one; the other is the six modules of the daughter platform have high similarity with the seven modules of the mother one. That is, besides the "Cultivating Community" module, the daughter platform has the same six modules of the mother one.

Their independence of each other mainly displays in three aspects. Firstly, the modules of "Introduction to the Platform" and "Medical Practice and Education Dynamic", which focus on introducing the technical platform of the cultivating community and relevant medical practice and education dynamic, have broader extension than those of the daughter one. Secondly, the modules of "Resource Sharing", "Distance Teaching", "Research Interaction" and "Planning Management" of the mother platform are designed with an emphasis different from those of the daughter one. The former provide experience and entrance for users, designed to manage the platform on the macro level while the latter are designed to provide users with specific services such as resource sharing, remote teaching, research interaction services and other planning management services on the micro level. Thirdly, the daughter platform can be independent of the mother one and directly accessed from the entry of each unit inside the cultivating community.

\section{Technical Support for Collaborative Training Platform}

Effective implementation of the platform mainly depends on multi-level distribution network system structure on macro level, predominant distributed architecture technology on median level and Web, multimedia and database technologies on micro level.

System Structure. Function diversity and user universality of the collaborative cultivating platform determine the adoption of a typical multilayer distributed network system structure. The platform is divided into four layers, i.e., user, web, application and data ones. Data support for each application module in the platform all comes from the data center. All users can access the platform through a Web browser and easily browse the various resources within the platform. Managers of the mother and daughter platforms through the web layer carry out functions such as data management, data maintenance, teacher and student management, statistical analysis, etc. Teachers and students can use the client software to achieve resource sharing, research interaction, man-machine information interactive teaching, two-way real-time multimedia conference teaching and other functions.

Architecture Technique. Distributed technology is key to the implementation of the platform, which is a kind of computer processing technology based on network and has a high degree of cohesiveness and transparency. In a distributed system, a set of independent computers display a unified picture to users. Therefore, for the users, a distributed system has only one model or 
paradigm. It has a wide range of physical and logical resources in common use and can dynamically allocate tasks, whose scattered physical and logical resources can realize information exchange through computer network. Therefore, distributed operating system employs a global mode to manage system resources and it can make servers distributed in various parts of the world so as to provide users with vast, convenient and fast service.

Technologies Employed. First is web technology. Web page is not only the most widely used human-computer interaction interface on Internet nowadays, but also an important channel for users to interact the collaborative cultivation platform. Dynamic web pages structured by XML, JavaScript, Ajax and JSP technologies provide users with a better man-machine interaction environment, and greatly improve the platform's usability and universality. Among them, Ajax (short form of Asynchronous JavaScript and XML) brings an interactive Web application technology able to enhance users' Web experience in the most direct way. However, JSP (short form of Java Server Pages) as a Web application of Java language is important guarantee for the implementation of dynamic Web pages on the server side. Moreover, JSP inherits the characteristic of cross-platform pertinent to Java language. This provides a strong support for the distributed deployment of the platform.

Second is multimedia technology. As the most direct and effective way in terms of users' senses, multimedia has an unshakable important status in computer-aided instruction. In distance education, however, there has always been irreconcilable conflict between images (acoustics) of excellent quality and the limited bandwidth and complicated network environment. The latest video coding technology $\mathrm{H} 264$ has brought us excellent picture quality, high compression ratio as well as strong network adaptability, enabling us to finding a dynamic balance point in the conflict. It has brought brand new remote teaching experience for users. Last but not least, strong support of Flash on streaming media makes us handy with facility in non-interactive remote teaching and VOD (short form of video on-demand).

Third is database technology. A safe, stable and efficient data center is the basic premise of the platform's effective operation. The functions and characteristics of the platform determine its data featuring massiveness and complexity. Database cluster technology, characteristic of "full scalability, high availability, high performance, excellent price performance ratio and resource sharing [5]", provides not only important guarantee for the platform's safe, stable and efficient operation, but a good support for massive and complex data processing. In numerous database products, Oracle database with its stability, complete function and good after-sales service become the best choice to serve as the data center of the collaborative cultivating technical platform.

\section{Acknowledgement}

This paper is a project funded by Science \& Technology Department of Sichuan Province (Name: Cultivation Mechanism Innovation Research of "Dual-track Combination" of Standardized Training of Resident Doctors and Postgraduates of Medical Professional Degree; No.: 14RKX0012); as well as a project receiving financial help from Postgraduate Education Reform and Innovation Funding (Name: Research on "System Normalization" Quality Guarantee System for Professional Degree Postgraduate Education of Local Medical Colleges of Sichuan Province; No: SCS14005). 


\section{Authors in brief}

Xiaoping ZHU, female, born in Lu County, master, associate research fellow Sichuan Province in 1977, chief of Training Section of Graduate College of Sichuan Medical University, whose research direction is graduate student education research and management;

Ke YU, male, born in 1971, research follow, director of Academic Affairs Division of Clinical Medical College of Sichuan Medical University, whose research direction is higher education research;

Gaofei CAO, male, born in 1978, bachelor, lecturer at Modern Education Technology Center of Sichuan Medical University, whose research direction is computer application technology.

\section{References}

[1] Information on http://www.moe.edu.cn/publicfiles/business/htmlfiles/moe/s7065/201411/178 832.html

[2] J. J. Feng, Research on the "track one" mode of clinical postgraduate education and standardized training of residencies, Chinese Medicine Modern Distance Education of China. 17, 12 (2014) 70-77.

[3] L. Chen, X. G. Yuan, Research on the construction of the technical platform of vocational education development under the background of urban-rural integration, E-education Research. 9 (2010) 98-101.

[4] X. R. Gou, B. Yu, Technical strategy to establishing modern distance education technology platform, Telecom Engineering Technics and Standardization. 3 (2000) 4-6.

[5] Y. B. Zhou, Research and implementation on common share-nothing database cluster, Computer Engineering and Applications. 32, 43 (2007) 156-160. 